\title{
Ricci Curvature for Warped Product Submanifolds of Sasakian Space Forms and Its Applications to Differential Equations
}

\author{
Fatemah Mofarreh $\left(\mathbb{D},{ }^{1}\right.$ Akram Ali $\mathbb{D}^{2},{ }^{2}$ Nadia Alluhaibi $\mathbb{D},{ }^{3}$ and Olga Belova $\mathbb{D}^{4}$ \\ ${ }^{1}$ Mathematical Science Department, Faculty of Science, Princess Nourah Bint Abdulrahman University, \\ Riyadh 11546, Saudi Arabia \\ ${ }^{2}$ Department of Mathematics, College of Science, King Khalid University, 9004 Abha, Saudi Arabia \\ ${ }^{3}$ Department of Mathematics, Science and Arts College, Rabigh Campus, King Abdulaziz University, Jeddah 21589, Saudi Arabia \\ ${ }^{4}$ Institute of Physical and Mathematical Sciences and IT, Immanuel Kant Baltic Federal University, 5A. Nevskogo st. 14, \\ 236016 Kaliningrad, Russia
}

Correspondence should be addressed to Fatemah Mofarreh; fyalmofarrah@pnu.edu.sa

Received 24 August 2020; Revised 6 November 2020; Accepted 22 December 2020; Published 15 January 2021

Academic Editor: Hang Xu

Copyright (c) 2021 Fatemah Mofarreh et al. This is an open access article distributed under the Creative Commons Attribution License, which permits unrestricted use, distribution, and reproduction in any medium, provided the original work is properly cited.

\begin{abstract}
In the present paper, we establish a Chen-Ricci inequality for a C-totally real warped product submanifold $M^{n}$ of Sasakian space forms $\mathbb{M}^{2 m+1}(\varepsilon)$. As Chen-Ricci inequality applications, we found the characterization of the base of the warped product $M^{n}$ via the first eigenvalue of Laplace-Beltrami operator defined on the warping function and a second-order ordinary differential equation. We find the necessary conditions for a base $\mathbb{B}$ of a $\mathrm{C}$-totally real-warped product submanifold to be an isometric to the Euclidean sphere $\mathbb{S}^{p}$.
\end{abstract}

\section{Introduction and Motivations}

For geometric analysis, the work of Obata [1] becomes an essential tool of investigation. Obata [1] provided a characterization theorem for a standard sphere in terms of a differential equation, known as the Obata equation. If $\left(M^{n}, g\right)$ is a complete manifold with $n \geq 1$, then the function $\varphi$ is nonconstant and fulfills the ordinary differential equation:

$$
\begin{array}{r}
\operatorname{Hess}(\varphi)+c \varphi g=0, \\
\operatorname{or} \nabla^{2} \varphi+c \varphi g=0,
\end{array}
$$

if and only if there is an isometry between $\left(M^{n}, g\right)$ and the sphere $\mathbb{S}^{n}(c)$, where $c$ denotes the sectional curvature. If $c=1$, then $\left(M^{n}, g\right)$ and the unit sphere $\mathbb{S}^{n}$ are congruent. A large number of investigations on this subject are studied. Therefore, the characterization of these spaces the Euclidean space $\mathbb{R}^{n}$, the Euclidean sphere $\mathbb{S}^{n}$, and the complex projective space $\mathbb{C} P^{n}$ are recognized fields in the study of differential geometry and are studied in research works such as [2-18]. In particular, the Euclidean space $\mathbb{R}^{n}$ is designated through the differential equation $\nabla^{2} \varphi=c g$, where $c$ is a positive constant, which is proven by Tashiro [19]. In [20], Lichnerowicz has established that if the first nonzero eigenvalue $\lambda_{1}$ of the Laplace operator of the compact manifold $\left(M^{n}, g\right)$ with Ric $\geq n-1$ is $\lambda_{1}=n$, then $\left(M^{n}, g\right)$ is isometric to the sphere $\mathbb{S}^{n}$. Thus, Obata's theorem can be utilized to address Lichnerowicz's eigenvalue equality condition derived in [20]. Deshmukh and Al-Solamy [21] proved that an $n$-dimensional connected Riemannian manifold $\left(M^{n}, g\right)$ which is compact and has a Ricci curvature satisfying $0<\operatorname{Ric} \leq(n-1)\left(2-\left(n c / \lambda_{1}\right) c\right)$ for a constant $c$, where $\lambda_{1}$ is the first eigenvalue of the Laplacian, is isometric to $\mathbb{S}^{n}(c)$ if $M^{n}$ is allowed to be a nonzero conformal gradient vector field. The authors also proved that if $M^{n}$ is an Einstein manifold, meaning that the Einstein constant is $\mu=(n-1) c$, then $M^{n}$ is isometric to $\mathbb{S}^{n}(c)$ with $c>0$ if a conformal gradient vector field is allowed. Taking account of ODE (1), Barros et al. [6] showed that the gradient of an almost Ricci 
soliton $\left(M^{n}, g, \nabla \varphi, \lambda\right)$ that is compact is isometric to the Euclidean sphere when the Ricci tensor is Codazzi, and a constant sectional curvature is present. For more information regarding the Obata equation, see [1]. Motivated by the previous studies, we establish a number of results in the present paper which realize as characterizations of spheres. More precisely, we have the following.

Theorem 1. Assume that $\Psi: M^{n}=\mathbb{B} \times{ }_{f} \mathbb{F} \longrightarrow \mathbb{M}^{2 m+1}(\varepsilon)$ is a $C$-totally real isometric embedding from a warped product submanifold $M^{n}$ into a Sasakian space form $\mathbb{M}^{2 m+1}(\varepsilon)$ with a nonnegative Ricci curvature. Then, the compact and minimal base $\mathbb{B}$ is isometric to the Euclidean sphere $\mathbb{S}^{p}\left(\sqrt{\lambda_{1} / p}\right)$ if the following equality holds:

$$
|\operatorname{Hess}(\varphi)|^{2}=\frac{\lambda_{1}}{4 p q}\left\{(\varepsilon+3)(1-n-p q)-n^{2}|\mathbb{H}|^{2}\right\},
$$

where $\lambda_{1}>0$ is the eigenvalue connected to an eigenfunction $\varphi=\ln ; f$ for the Laplacian operator and Hess $(\varphi)$ is a Hessian tensor for the function $\varphi$. Moreover, here, the constant curvature $c$ is equal to $\sqrt{\lambda_{1} / p}$. In a particular case, if $\lambda_{1}=p$ satisfies the condition

$$
|\operatorname{Hess}(\varphi)|^{2}=\frac{1}{4 q}\left\{(\varepsilon+3)\left(1-n-\lambda_{1} q\right)-n^{2}|\mathbb{H}|^{2}\right\}
$$

then, the base $\mathbb{B}$ is isometric to the standard sphere $\mathbb{S}^{p}$.

From the Bochner formula, we are able to prove the following result:

Theorem 2. Let $\Psi: M^{n}=\mathbb{B} \times{ }_{f} \mathbb{F} \longrightarrow \mathbb{M}^{2 m+1}(\varepsilon)$ be a C-totally real isometric embedding from a warped product submanifold $M^{n}$ into a Sasakian space form $\mathbb{M}^{2 m+1}(\varepsilon)$ having the nonnegative Ricci curvature. Then, compact and minimal base $\mathbb{B}$ is isometric to the sphere $\mathbb{S}^{p}(c)$ with a constant sectional curvature equal to $c=\sqrt{\lambda_{1} / p}$ if the following equality holds:

$$
|\mathbb{H}|^{2}=\frac{(\varepsilon+3)(1-p q-n)}{n^{2}},
$$

where $\lambda_{1}>0$ is a positive eigenvalue associated with the eigenfunction $\varphi=\ln ; f$. Moreover, $n=\operatorname{dim} M, p=\operatorname{dim} \mathbb{B}$, and $q=\operatorname{dim} \mathbb{F}$.

The paper is organized as follows. In Section 2, we study some preliminaries formulas, notations, and definitions related to our study. In the same section, we prove a lemma, a key result for our main theorem. In Section 3, we demonstrate our main conclusions and provide several consequences from our main findings. We also give an example for existence $C$-totally real warped product submanifold in Sasakian manifolds. In Section 4, we give concluding remarks.

\section{Preliminaries and Notations}

Let $(\tilde{\mathbb{M}}, t g)$ be the odd-dimensional $C^{\infty}$-manifold equipped with an almost contact structure $(\psi, \zeta, \eta)$ such that

$$
\begin{aligned}
\psi^{2} & =-I+\eta \otimes \zeta, \quad \eta(\zeta)=1, \psi(\zeta)=0, \eta \psi=0, \\
g\left(\psi W_{1}, \psi W_{2}\right) & =g\left(W_{1}, W_{2}\right)-\eta\left(W_{1}\right) \eta\left(W_{2}\right), \eta\left(W_{1}\right) \\
& =g\left(W_{1}, \zeta\right),
\end{aligned}
$$

for any $W_{1}, W_{2} \in \Gamma(T \tilde{M})$. Of course, the notations are well known: $\zeta$ is a structure vector, the $(1,1)$-type tensor field is denoted by $\psi$, and $\eta$ is the dual one-form. Moreover, the tensorial equation for a Sasakian manifold [22-24] with the structure $(\psi, \zeta, \eta)$ is given by

$$
\left(\widetilde{\nabla}_{W_{1}} \psi\right) W_{2}=g\left(W_{1}, W_{2}\right) \zeta-\eta\left(W_{2}\right) W_{1}, \widetilde{\nabla}_{W_{1}} \zeta=-\psi W_{1} .
$$

If we choose two vector fields $W_{1}$ and $W_{2}$ at $\tilde{\mathbb{M}}$, such that $\tilde{\nabla}$ is the Riemannian connection regarding $g$, and assume that $\widetilde{\mathbb{M}}^{2 m+1}(\varepsilon)$ is a Sasakian space form with a constant $\psi$-sectional curvature $\varepsilon$, then its curvature tensor $\widetilde{R}$ is

$$
\begin{aligned}
\widetilde{R}\left(W_{1}, W_{2}, W_{3}, W_{4}\right)= & \frac{\varepsilon+3}{4}\left\{g\left(W_{2}, W_{3}\right) g\left(W_{1}, W_{4}\right)-g\left(W_{1}, W_{3}\right) g\left(W_{2}, W_{4}\right)\right\} \\
& +\frac{\varepsilon-1}{4}\left\{\eta\left(W_{1}\right) \eta\left(W_{3}\right) g\left(W_{2}, W_{4}\right)+\eta\left(W_{4}\right) \eta\left(W_{2}\right) g\left(W_{1}, W_{3}\right)\right. \\
& -\eta\left(W_{2}\right) \eta\left(W_{3}\right) g\left(W_{1}, W_{4}\right)-\eta\left(W_{1}\right) g\left(W_{2}, W_{3}\right) \eta\left(W_{4}\right) \\
& +g\left(\psi W_{2}, W_{3}\right) g\left(\psi W_{1}, W_{4}\right)-g\left(\psi W_{1}, W_{3}\right) g\left(\psi W_{2}, W_{4}\right) \\
& \left.+2 g\left(W_{1}, \psi W_{2}\right) g\left(\psi W_{3}, W_{4}\right)\right\},
\end{aligned}
$$

for all $W_{1}, W_{2}, W_{3}, W_{4} \in \Gamma(T \tilde{M})$. The odd-dimensional Euclidean space $\mathbb{R}^{2 m+1}$ and odd-dimensional sphere $\mathbb{S}^{2 m+1}$ with sectional curvatures of $\varepsilon=-3$ and $\varepsilon=1$ are remarkable examples of Sasakian space forms in [25]. Moreover, if the structure vector field $\zeta$ belongs to the normal space of $M^{n}$, then $M^{n}$ is said to be a C-totally real submanifold; for more 
details, see the work presented in [22-24, 26, 27]. It should be noted that the curvature tensor $\widetilde{R}$ for $M^{n}$ in Sasakian space form $\widetilde{M}^{2 m+1}(\varepsilon)$ is defined as

$$
\widetilde{R}\left(W_{1}, W_{2}, W_{3}, W_{4}\right)=\left(\frac{\varepsilon+3}{4}\right)\left\{g\left(W_{2}, W_{3}\right) g\left(W_{1}, W_{4}\right)-g\left(W_{1}, W_{3}\right) g\left(W_{2}, W_{4}\right)\right\}
$$

Suppose $M^{n}$ is a Riemannian submanifold of a Riemannian manifold $\mathbb{M}^{2 m+1}$ considering the induced metric $g$ and $\nabla$ and $\nabla^{\perp}$ are connections along $T M$ and $T^{\perp} M$ of $M^{n}$, where $T M$ is a tangent bundle and $T^{\perp} M$ is a normal bundle of $M^{n}$. Therefore, for any $W_{1}, W_{2} \in \mathfrak{X}(T M)$ and $\xi \in \mathfrak{X}\left(T^{\perp} M\right)$, the Gauss and Weingarten formulas are written as $\quad \tilde{\nabla}_{W_{1}} W_{2}=\nabla_{W_{1}} W_{2}+\mathbf{B}\left(W_{1}, W_{2}\right)$ and $\tilde{\nabla}$ ${ }_{W_{1}} \xi=-A_{\xi} W_{1}+\nabla_{W_{1}}^{\perp} \xi$, respectively. Note that $\mathbf{B}$ and $A_{\xi}$ denote the second fundamental form as well as the shape operator, respectively. They are governed by the relation $g\left(\mathbf{B}\left(W_{1}, W_{2}\right), N\right)=g\left(A_{\xi} W_{1}, W_{2}\right)$. The Gauss equation is

$$
R\left(W_{1}, W_{2}, W_{3}, W_{4}\right)=\widetilde{R}\left(W_{1}, W_{2}, W_{3}, W_{4}\right)+g\left(\mathbf{B}\left(W_{1}, W_{4}\right), \mathbf{B}\left(W_{2}, W_{3}\right)\right)-g\left(\mathbf{B}\left(W_{1}, W_{3}\right), \mathbf{B}\left(W_{2}, W_{4}\right)\right),
$$

for any $W_{1}, W_{2}, W_{3}, W_{4} \in \mathfrak{X}(\tilde{M})$, where the curvature tensors of $\mathbb{M}^{2 m}+1$ and $M^{n}$ are represented by $\widetilde{R}$ and $R$. Furthermore, $H$, which is the mean curvature of $M^{n}$, is calculated as $H=(1 / n) \operatorname{trace}(\mathbf{B}) . M^{n}$ is totally umbilical if $\mathbf{B}\left(W_{1}, W_{2}\right)=g\left(W_{1}, W_{2}\right) H$ and totally geodesic if $\mathbf{B}\left(W_{1}, W_{2}\right)=0$, for any $W_{1}, W_{2} \in \mathfrak{X}(M)$. Furthermore, $M^{n}$ is minimal if $H=0$. Here,

$$
\mathcal{N}_{x}=\left\{X \in T_{x} M \mid \mathbf{B}\left(W_{1}, W_{2}\right)=0, \text { for all } W_{2} \in T_{x} M\right\}
$$

gives the second fundamental form kernel of $M^{n}$ over $x$. If the plane section is spanned by $e_{\alpha}$ and $e_{\beta}$ over $x$ in $\tilde{M}^{2 m+1}$, then such a curvature is called a sectional curvature and is denoted by $\widetilde{K}_{\alpha \beta}=\widetilde{K}\left(e_{\alpha} \wedge e_{\beta}\right)$. The relation between the scalar curvature $\widetilde{\tau}\left(T_{x} \tilde{M}\right)$ of $\mathbb{M}^{2 m+1}$ and $\widetilde{K}\left(e_{\alpha} \wedge e_{\beta}\right)$ at some $x$ in $\mathbb{M}^{2 m+1}$ is represented by

$$
\tilde{\tau}\left(T_{x} \tilde{M}\right)=\sum_{1 \leq \alpha<\beta \leq 2 m+1} \widetilde{K}_{\alpha \beta} .
$$

The first equality in (11) is reciprocal to the following:

$$
2 \widetilde{\tau}\left(T_{x} M^{n}\right)=\sum_{1 \leq \alpha<\beta \leq n} \widetilde{K}_{\alpha \beta}, \quad 1 \leq \alpha, \beta \leq n .
$$

The previous relation will be utilized in the subsequent proofs. Similarly, the scalar curvature $\tilde{\tau}\left(L_{x}\right)$ of an $L$-plan is expressed as

$$
\tilde{\tau}\left(L_{x}\right)=\sum_{1 \leq \alpha<\beta \leq m} \widetilde{K}_{\alpha \beta} .
$$

Let $\left\{e_{1}, \ldots, e_{n}\right\}$ be an orthonormal frame of the tangent space $T_{x} M$ and $e_{r}=\left(e_{n+1}, \ldots, e_{2 m+1}\right)$ be an orthonormal frame of the normal space $T^{\perp} M$. Thus, we have

$$
\begin{aligned}
\mathbf{B}_{\alpha \beta}^{r} & =g\left(\mathbf{B}\left(e_{\alpha}, e_{\beta}\right), e_{r}\right), \\
\|\mathbf{B}\|^{2} & =\sum_{\alpha, \beta=1}^{n} g\left(\mathbf{B}\left(e_{\alpha}, e_{\beta}\right), \mathbf{B}\left(e_{\alpha}, e_{\beta}\right)\right)=\sum_{\alpha, \beta=1}^{n}\left(\mathbf{B}_{\alpha \beta}^{r}\right)^{2} .
\end{aligned}
$$

Let $K_{\alpha \beta}$ and $\widetilde{K}_{\alpha \beta}$ be the sectional curvature of a submanifold $M^{n}$ and $\mathbb{M}^{2 m+1}$; then, we have following relation due to the Gauss equation (9):

$$
\begin{aligned}
2 \tau\left(T_{x} M^{n}\right) & =K_{\alpha \beta}=2 \widetilde{\tau}\left(T_{x} M^{n}\right)+\sum_{r=n+1}^{2 m+1}\left(\mathbf{B}_{\alpha \alpha}^{r} \mathbf{B}_{\beta \beta}^{r}-\left(\mathbf{B}_{\alpha \beta}^{r}\right)^{2}\right) \\
& =\widetilde{K}_{\alpha \beta}+\sum_{r=n+1}^{2 m+1}\left(\mathbf{B}_{\alpha \alpha}^{r} \mathbf{B}_{\beta \beta}^{r}-\left(\mathbf{B}_{\alpha \beta}^{r}\right)^{2}\right) .
\end{aligned}
$$

Furthermore, the Ricci tensor is defined as

$$
\widetilde{S}\left(W_{1}, W_{2}\right)=\sum_{i=1}^{n}\left\{\widetilde{g}\left(\widetilde{R}\left(e_{\alpha}, W_{1}\right) W_{2} e_{\alpha}\right)\right\}, \quad W_{1}, W_{2} \in \Gamma\left(T_{x} M^{n}\right) .
$$

Fixing the distinct indices for vector fields from $\left\{e_{1}, \ldots, e_{n}\right\}$ on $M^{n}$ by $e_{u}$, which is governed by $W$, the Ricci curvature is given as

$$
\operatorname{Ric}(W)=\sum_{\substack{\alpha=1 \\ \alpha \neq u}}^{n} K\left(e_{\alpha} \wedge e_{u}\right)
$$


Therefore, equation (12) can be written as

$$
\tilde{\tau}\left(T_{x} M^{n}\right)=\sum_{1 \leq \alpha<\beta \leq n} K\left(e_{\alpha} \wedge e_{\beta}\right)=\frac{1}{2} \sum_{A=1}^{n} \operatorname{Ric}\left(e_{u}\right) .
$$

Thus,

$$
2 \widetilde{\tau}\left(T_{x} M^{n}\right)=\sum_{1 \leq \alpha<\beta \leq n} K\left(e_{\alpha} \wedge e_{\beta}\right)=\frac{1}{2} \sum_{u=1}^{n} \operatorname{Ric}\left(e_{u}\right),
$$

which will be frequently used in future studies. The gradientsquared norm of the positive smooth function $\varphi$ for an orthonormal basis $\left\{e_{1}, \ldots, e_{n}\right\}$ is given by

$$
\|\nabla \varphi\|^{2}=\sum_{i=1}^{n}\left(e_{i}(\varphi)\right)^{2} .
$$

Assume that $\mathbb{B}$ and $\mathbb{F}$ are Riemannian manifolds with Riemannian metrics $g_{1}$ and $g_{2}$, respectively. Suppose $f$ is a differentiable function on $\mathbb{B}$. Then, the manifold $\mathbb{B} \times \mathbb{F}$ endorsed by the Riemannian metric $g=g_{1}+f^{2} g_{2}$ is referred to a warped product manifold and classified as notation $M^{n}=\mathbb{B} \times_{f} \mathbb{F}[28]$. Assume that $M^{n}=\mathbb{B} \times_{f} \mathbb{F}$ is a warped product, then we have

$$
\nabla_{W_{2}} W_{1}=\nabla_{W_{1}} W_{2}=\left(W_{1} \ln f\right) W_{2}
$$

$\forall W_{1} \in \Gamma(\mathbb{B})$ and $W_{2} \in \Gamma(\mathbb{F})$. It was proved in Section 3.3 in [28] that the following relation holds:

$$
\sum_{\alpha=1}^{p} \sum_{\beta=1}^{q} K\left(e_{\alpha} \wedge e_{\beta}\right)=\frac{q \Delta f}{f}=q\left(\Delta(\ln f)-\|\nabla(\ln f)\|^{2}\right) .
$$

Remark 1. $M^{n}=\mathbb{B} \times{ }_{f} \mathbb{F}$ is Riemannian product manifold if $f$ is a constant.

Remark 2. Sometimes we will use the following abbreviation throughout the paper: "WPS" for Warped product submanifold, "WF" for warping function, "RM" for Riemannian manifold, and "SSF" for Sasakian space form.

\section{Ricci Curvature for C-Totally Real Warped Products}

Inspired by the work [2, 3, 9], we prove the following proposition which we will use in further result.

Proposition 1. Let $M^{n}=\mathbb{B} \times_{f} \mathbb{F}$ be a C-totally real warped product submanifold into a Sasakian space form $\mathbb{M}^{2 m+1}(\varepsilon)$ having the minimal base $\mathbb{B}$. Then, for all unit vectors $W \in T_{x} M^{n}$, the following Ricci inequality holds:

$$
\mathscr{R} i c(W)+q \Delta \ln f \leq \frac{n^{2}}{4}\|H\|^{2}+q\|\nabla \ln f\|^{2}+\frac{\varepsilon+3}{4}(p q+n-1)
$$

where $p=\operatorname{dim} \mathbb{B}$ and $q=\operatorname{dim} \mathbb{F}$.

(i) In case $H(x)=0$, for $x \in M^{n}$, there exists a unit vector $W$ satisfying the equality in (23) if and only if $M^{n}$ is mixed totally geodesic and $W$ lies in $\mathcal{N}_{x}$ at $x$.

(ii) If $M^{n}$ is $\mathbb{B}$-minimal, thus

(a) The equality in (23) remains for any unit tangent vectors at $\mathbb{B}$ and any $x \in M^{n} \longrightarrow M^{n}$ is totally geodesic and $\mathbb{B}$-totally geodesic WPS in $\mathbb{M}^{2 m+1}(\varepsilon)$.

(b) The equality in (23) remains for any unit tangent vectors at $\mathbb{F}$ and any $x \in M^{n} \longrightarrow M^{n}$ is totally geodesic, either a F-totally geodesic WPS or a F-totally umbilical WPS in $\mathbb{M}^{2 m+1}(\varepsilon)$ such that $\operatorname{dim} \mathbb{F}=2$.

(iii) The equality in (23) is satisfied for any unit tangent vectors at $M^{n}$ and any $x \in M^{n} \longrightarrow M^{n}$ is either totally geodesic, or totally umbilical, mixed totally geodesic, and $\mathbb{B}$-totally geodesic WPS such that $\operatorname{dim} \mathbb{F}=2$.

Proof. Assume that $M^{n}$ is a $\mathbb{B}$-minimal C-totally real warped product. An analogous technique will be used for similar cases. Utilizing the Gauss equation (9), we derive

$$
n^{2}\|\mathbb{H}\|^{2}=2 \tau\left(T_{x} M^{n}\right)+\|\mathbf{B}\|^{2}-2 \widetilde{\tau}\left(T_{x} M^{n}\right) .
$$

Assume $\left\{e_{1}, \ldots, e_{p}, e_{p+1}, \ldots, e_{n}\right\}$ is the local orthonormal frame field of $\mathbb{M}^{2 m+1}(\varepsilon)$ in which the basis $\left\{e_{1}, \ldots, e_{p}\right\}$ are tangent to $\mathbb{B}$ and $\left\{e_{p+1}, \ldots, e_{n}\right\}$ are tangent to $\mathbb{F}$. Thus, for the unit tangent vector $W=e_{u} \in\left\{e_{1}, \ldots, e_{n}\right\}$, we can expand (24):

$$
n^{2}\|\mathbb{H}\|^{2}=2 \tau\left(T_{x} M^{n}\right)+\frac{1}{2} \sum_{r=n+1}^{2 m+1}\left\{\left(\mathbf{B}_{11}^{r}+\cdots+\mathbf{B}_{n n}^{r}-\mathbf{B}_{u u}^{r}\right)^{2}+\left(\mathbf{B}_{u u}^{r}\right)^{2}\right\}-\sum_{r=n+1}^{2 m+1} \sum_{1 \leq \alpha \neq \beta \leq n} \mathbf{B}_{\alpha \alpha}^{r} \mathbf{B}_{\beta \beta}^{r}-2 \widetilde{\tau}\left(T_{x} M^{n}\right),
$$


which is equivalent to the following by using (8):

$$
\begin{aligned}
n^{2}\|\mathbb{E}\|^{2}= & 2 \tau\left(T_{x} M^{n}\right)+\sum_{r=n+1}^{2 m+1}\left\{\left(\mathbf{B}_{11}^{r}+\cdots+\mathbf{B}_{n n}^{r}\right)^{2}+\left(2 \mathbf{B}_{u u}^{r}-\left(\mathbf{B}_{11}^{r}+\cdots+\mathbf{B}_{n n}^{r}\right)\right)^{2}\right\} \\
& +2 \sum_{r=n+1}^{2 m+1} \sum_{1 \leq \alpha<\beta \leq n}\left(\mathbf{B}_{\alpha \beta}^{r}\right)^{2}-2 \sum_{r=n+1}^{2 m+1} \sum_{1 \leq \alpha<\beta \leq n} \mathbf{B}_{\alpha}^{r} \mathbf{B}_{\beta}^{r}-\frac{\varepsilon+3}{4} n(n-1) .
\end{aligned}
$$

As we assumed that the base of the warped product submanifold $M^{n}$ is minimal, we derive

$$
\begin{aligned}
& n^{2}\|\mathbb{E}\|^{2}+\frac{\varepsilon+3}{4} n(n-1)=\sum_{r=n+1}^{2 m+1}\left\{\left(\mathbf{B}_{p+1 n_{1}+1}^{r}+\cdots+\mathbf{B}_{n n}^{r}\right)^{2}+\left(2 \mathbf{B}_{u u}^{r}-\left(\mathbf{B}_{p+1 p+1}^{r}+\cdots+\mathbf{B}_{n n}^{r}\right)\right)^{2}\right\} \\
& +2 \tau\left(T_{x} M^{n}\right)+\sum_{r=n+1}^{2 m+1} \sum_{1 \leq \alpha<\beta \leq n}\left(\mathbf{B}_{\alpha \beta}^{r}\right)^{2}-\sum_{r=n+1}^{2 m+1} \sum_{1 \leq \alpha<\beta \leq n} \mathbf{B}_{\alpha \alpha}^{r} \mathbf{B}_{\beta \beta}^{r} \\
& +\sum_{r=n+1}^{2 m+1} \sum_{a=1 a \neq u}\left(\mathbf{B}_{a u}^{r}\right)^{2}+\sum_{r=n+1}^{m} \sum_{\substack{1 \leq \alpha<\beta \leq n \\
\alpha, \beta \neq u}}\left(\mathbf{B}_{\alpha \beta}^{r}\right)^{2}-\sum_{r=n+1}^{2 m+1} \sum_{\substack{1 \leq \alpha<\beta \leq n \\
\alpha, \beta \neq u}} \mathbf{B}_{\alpha \alpha}^{r} \mathbf{B}_{\beta \beta}^{r} .
\end{aligned}
$$

In view of (15), we obtain

$$
\sum_{r=n+1}^{2 m+1} \sum_{\substack{1 \leq \alpha<\beta \leq n \\ \alpha, \beta \neq u}}\left(\mathbf{B}_{\alpha \beta}^{r}\right)^{2}-\sum_{r=n+1}^{2 m+1} \sum_{\substack{1 \leq \alpha<\beta \leq n \\ \alpha, \beta \neq u}} \mathbf{B}_{\alpha \alpha}^{r} \mathbf{B}_{\beta \beta}^{r}=\sum_{\substack{1 \leq \alpha<\beta \leq n \\ \alpha, \beta \neq A}} \widetilde{K}_{\alpha \beta}-\sum_{\substack{1 \leq \alpha<\beta \leq n \\ \alpha, \beta \neq A}} K_{\alpha \beta} .
$$

From the fact that the base $\mathbb{B}$ is minimal and putting (28) in (27), we deduce

$$
\begin{aligned}
& \frac{1}{2} n^{2}\|\mathbb{H}\|^{2}+\frac{\varepsilon+3}{4} n(n-1)=2 \tau\left(T_{x} M^{n}\right)+\frac{1}{2} \sum_{r=n+1}^{2 m+1}\left(2 \mathbf{B}_{u u}^{r}-\left(\mathbf{B}_{p+1 p+1}^{r}+\cdots+\mathbf{B}_{n n}^{r}\right)\right)^{2} \\
& +\sum_{r=n+1}^{2 m+1} \sum_{1 \leq \alpha<\beta \leq n}\left(\mathbf{B}_{\alpha \beta}^{r}\right)^{2}-\sum_{r=n+1}^{m} \sum_{\substack{1 \leq \alpha<\beta \leq n \\
\alpha, \beta \neq u}} \mathbf{B}_{\alpha \alpha}^{r} \mathbf{B}_{\beta \beta}^{r}+\sum_{r=n+1}^{2 m+1} \sum_{\substack{a=1, a \neq u\\
}}\left(\mathbf{B}_{a u}^{r}\right)^{2}+\sum_{\substack{1 \leq \alpha<\beta \leq n \\
\alpha, \beta \neq u}} \widetilde{K}_{\alpha \beta}-\sum_{\substack{1 \leq \alpha<\beta \leq n \\
\alpha, \beta \neq u}} K_{\alpha \beta} .
\end{aligned}
$$


On the contrary, using (11), we define

$$
\begin{aligned}
\tau\left(T_{x} M^{n}\right) & =\sum_{1 \leq \alpha<\beta \leq n} K\left(e_{\alpha} \wedge e_{\beta}\right) \\
& =\sum_{i=1}^{p} \sum_{j=p+1}^{n} K\left(e_{i} \wedge e_{j}\right)+\sum_{1 \leq i<k \leq p} K\left(e_{i} \wedge e_{k}\right)+\sum_{p+1 \leq l<o \leq n} K\left(e_{l} \wedge e_{o}\right) .
\end{aligned}
$$

From (22) and (11), we obtain

From (29)-(31) and using (14), we deduce

$$
\tau\left(T_{x} M^{n}\right)=\frac{q \Delta f}{f}+\tau\left(T_{x} \mathbb{B}^{p}\right)+\tau\left(T_{x} \mathbb{F}^{q}\right) .
$$

$$
\begin{aligned}
& \frac{1}{2} n^{2}\|\mathbb{H}\|^{2}+\frac{\varepsilon+3}{4} n(n-1)=\frac{q \Delta f}{f}-2 \widetilde{\tau}\left(T_{x} M^{n}\right)+\sum_{\substack{1 \leq \alpha<\beta \leq n \\
\alpha, \beta \neq u}} \widetilde{K}_{\alpha \beta}+\widetilde{\tau}\left(T_{x} \mathbb{B}^{p}\right)+\widetilde{\tau}\left(T_{x} \mathbb{F}^{q}\right) \\
& +\sum_{r=n+1}^{2 m+1}\left\{\sum_{1 \leq \alpha<\beta \leq n}\left(\mathbf{B}_{\alpha \beta}^{r}\right)^{2}-\sum_{\substack{1 \leq \alpha<\beta \leq n \\
\alpha, \beta \neq u}} \mathbf{B}_{\alpha \alpha}^{r} \mathbf{B}_{\beta \beta}^{r}\right\}+\sum_{r=n+1}^{2 m+1} \sum_{\substack{a=1 \\
a \neq u}}\left(\mathbf{B}_{a u}^{r}\right)^{2}+\sum_{r=n+1}^{m} \sum_{\substack{1 \leq i \neq j \leq p\\
}}\left(\mathbf{B}_{i i}^{r} \mathbf{B}_{j j}^{r}-\left(\mathbf{B}_{i j}^{r}\right)^{2}\right) \\
& +\sum_{r=n+1}^{2 m+1} \sum_{p+1 \leq s \neq t \leq n}\left(\mathbf{B}_{s s}^{r} \mathbf{B}_{t t}^{r}-\left(\mathbf{B}_{s t}^{r}\right)^{2}\right)+\frac{1}{2} \sum_{r=n+1}^{2 m+1}\left(2 \mathbf{B}_{u u}^{r}-\left(\mathbf{B}_{p+1 p+1}^{r}+\cdots+\mathbf{B}_{n n}^{r}\right)\right)^{2} .
\end{aligned}
$$

Now, we note that $e_{u}$ is either tangent to the base $\mathbb{B}$ or to the fiber $\mathbb{F}$. After that, the proof of the former case is introduced.
Case 1. Let $e_{u}$ be tangent to $\mathbb{B}$. We fix the unit tangent vector from $\left\{e_{1}, \ldots, e_{p}\right\}$ to be $e_{u}$ and consider $W=e_{u}=e_{1}$. Then, from (17) and (32), we obtain

$$
\begin{aligned}
\frac{1}{2} n^{2}\|\mathbb{H}\|^{2} \geq & \mathscr{R} i c(W)+\frac{q \Delta f}{f}-2 \widetilde{\tau}\left(T_{x} M^{n}\right)+\widetilde{\tau}\left(T_{x} \mathbb{B}^{p}\right)+\widetilde{\tau}\left(T_{x} \mathbb{F}\right)-\frac{\varepsilon+3}{4} n(n-1) \\
& +\sum_{2 \leq \alpha<\beta \leq n} \widetilde{K}_{\alpha \beta}+\frac{1}{2} \sum_{r=n+1}^{2 m+1}\left(2 \mathbf{B}_{11}^{r}-\left(\mathbf{B}_{p+1 p+1}^{r}+\cdots+\mathbf{B}_{n n}^{r}\right)\right)^{2} \\
& +\sum_{r=n+1}^{2 m+1} \sum_{1 \leq \alpha<\beta \leq n}\left(\mathbf{B}_{\alpha \beta}^{r}\right)^{2}-\sum_{r=n+1}^{2 m+1}\left\{\sum_{1 \leq i<j \leq p}\left(\mathbf{B}_{i j}^{r}\right)^{2}+\sum_{p+1 \leq s<t \leq n}\left(\mathbf{B}_{s t}^{r}\right)^{2}\right\} \\
& +\sum_{r=n+1}^{2 m+1}\left\{\sum_{1 \leq i<j \leq p} \mathbf{B}_{i i}^{r} \mathbf{B}_{j j}^{r}+\sum_{r=n+1}^{2 m+1} \sum_{p+1 \leq s \neq t \leq n} \mathbf{B}_{s s}^{r} \mathbf{B}_{t t}^{r}-\sum_{2 \leq \alpha<\beta \leq n} \mathbf{B}_{\alpha \alpha}^{r} h_{\beta \beta}^{r}\right\} .
\end{aligned}
$$

Substituting $W_{1}=W_{2}=e_{\alpha}$ and $W_{2}=W_{2}=e_{\beta}$ for $1 \leq \alpha, \beta \leq n$ in (7) and summarizing, we obtain

$$
\sum_{\alpha, \beta=1}^{n} \widetilde{R}\left(e_{\alpha}, e_{\beta}, e_{\alpha}, e_{\beta}\right)=\frac{\varepsilon+3}{4} n(n-1) .
$$


Therefore, using (34) in equation (33), we obtain

$$
\begin{aligned}
\mathscr{R} \mathcal{C}_{M}(W) \leq & \frac{n^{2}}{2}\|\mathbb{H}\|^{2}-\frac{q \Delta f}{f}+\frac{\varepsilon+3}{4}(p q+n-1) \\
& -\frac{1}{2} \sum_{r=n+1}^{m}\left(2 \mathbf{B}_{11}^{r}-\left(\mathbf{B}_{p+1 p+1}^{r}+\cdots+\mathbf{B}_{n n}^{r}\right)\right)^{2} \\
& +\sum_{r=n+1}^{2 m+1}\left\{\sum_{1 \leq i<j \leq p}\left(\mathbf{B}_{i j}^{r}\right)^{2}+\sum_{p+1 \leq s<t \leq n}\left(\mathbf{B}_{s t}^{r}\right)^{2}\right\} \\
& -\sum_{r=n+1}^{2 m+1}\left\{\sum_{1 \leq i<j \leq p} \mathbf{B}_{i i}^{r} \mathbf{B}_{j j}^{r}+\sum_{r=n+1}^{m} \sum_{p+1 \leq s \neq t \leq n} \mathbf{B}_{s s}^{r} \mathbf{B}_{t t}^{r}\right\} \\
& +\sum_{r=n+1}^{2 m+1} \sum_{2 \leq \alpha<\beta \leq n} \mathbf{B}_{\alpha \alpha}^{r} \mathbf{B}_{\beta \beta}^{r}-\sum_{r=n+1}^{2 m+1} \sum_{1 \leq \alpha<\beta \leq n}\left(\mathbf{B}_{\alpha \beta}^{r}\right)^{2} .
\end{aligned}
$$

The calculation of the last two terms of (35) implies

$$
\sum_{r=n+1}^{2 m+1}\left\{\sum_{1 \leq i<j \leq p}\left(\mathbf{B}_{i j}^{r}\right)^{2}+\sum_{p+1 \leq s<t \leq n}\left(\mathbf{B}_{s t}^{r}\right)^{2}\right\}-\sum_{r=n+1}^{2 m+1} \sum_{1 \leq \alpha<\beta \leq n}\left(\mathbf{B}_{\alpha \beta}^{r}\right)^{2}=\sum_{r=n+1}^{2 m+1} \sum_{\alpha=1}^{p} \sum_{\beta=p+1}^{n}\left(\mathbf{B}_{\alpha \beta}^{r}\right)^{2} .
$$

In similar way, we obtain

$$
\sum_{r=n+1}^{2 m+1}\left\{\sum_{1 \leq i<j \leq p} \mathbf{B}_{i i}^{r} \mathbf{B}_{j j}^{r}+\sum_{r=n+1}^{m} \sum_{p+1 \leq s \neq t \leq n} \mathbf{B}_{s s}^{r} \mathbf{B}_{t t}^{r}-\sum_{2 \leq \alpha<\beta \leq n} \mathbf{B}_{\alpha}^{r} \mathbf{B}_{\beta}^{r}\right\}=\sum_{r=n+1}^{2 m+1}\left(\sum_{j=2}^{p} \mathbf{B}_{11}^{r} \mathbf{B}_{j j}^{r}-\sum_{\alpha=2}^{p} \sum_{\beta=p+1}^{n} \mathbf{B}_{\alpha \alpha}^{r} \mathbf{B}_{\beta \beta}^{r}\right) .
$$

Using (37) in (33) leads to

$$
\begin{aligned}
\mathscr{R i c}(W) \leq & \frac{1}{2} n^{2}\|\mathbb{H}\|^{2}-\frac{q \Delta f}{f}+\frac{\varepsilon+3}{4}(p q+n-1) \\
& -\sum_{r=n+1}^{2 m+1}\left(\sum_{\alpha=1}^{p} \sum_{\beta=p+1}^{n}\left(\mathbf{B}_{\alpha \beta}\right)^{2}+\sum_{b=2}^{n_{1}} \mathbf{B}_{11}^{r} \mathbf{B}_{b b}^{2}-\sum_{\alpha=2}^{p} \sum_{\beta=p+1}^{n} \mathbf{B}_{\alpha \alpha}^{r} \mathbf{B}_{\beta \beta}^{r}\right) \\
& -\frac{1}{2} \sum_{r=n+1}^{m}\left(2 \mathbf{B}_{11}^{r}-\left(\mathbf{B}_{p+1 p+1}^{r}+\cdots+\mathbf{B}_{n n}^{r}\right)\right)^{2} .
\end{aligned}
$$

As for the warped product submanifold $M^{n}$ such that the base is minimal in $M^{n}$, we compute the following $\sum_{r=n+1}^{2 m+1} \sum_{\alpha=2}^{p} \sum_{\beta=p+1}^{n} \mathbf{B}_{\alpha \alpha}^{r} \mathbf{B}_{\beta \beta}^{r}=-\sum_{r=n+1}^{2 m+1} \sum_{\beta=p+1}^{n} \mathbf{B}_{11}^{r} \mathbf{B}_{\beta \beta}^{r}$. simplification: 
Similarly, we have

$$
\sum_{r=n+1}^{2 m+1} \sum_{b=2}^{p} \mathbf{B}_{11}^{r} \mathbf{B}_{b b}^{r}=-\sum_{r=n+1}^{2 m+1}\left(\mathbf{B}_{11}^{r}\right)^{2}
$$

At the same time, utilizing the minimality of the base manifold $\mathbb{B}^{p}$, we deduce that

$$
\begin{aligned}
& \frac{1}{2} \sum_{r=n+1}^{2 m+1}\left(2 \mathbf{B}_{11}^{r}-\left(\mathbf{B}_{p+1 p+1}^{r}+\cdots+\mathbf{B}_{n n}^{r}\right)\right)^{2}+\sum_{r=n+1}^{2 m+1} \sum_{\beta=p+1}^{n} \mathbf{B}_{11}^{r} \mathbf{B}_{\beta \beta}^{r} \\
& =2 \sum_{r=n+1}^{2 m+1}\left(\mathbf{B}_{11}^{r}\right)^{2}+\frac{1}{2} n^{2}\|\mathbb{U}\|^{2} .
\end{aligned}
$$

Utilizing (39)-(41), equation (38) will take the form

$$
\mathscr{R i c}(X) \leq \frac{\varepsilon+3}{4}(p q+n-1)-\frac{q \Delta f}{f}+\sum_{r=n+1}^{2 m+1}\left\{\sum_{\beta=p+1}^{n} \mathbf{B}_{11}^{r} \mathbf{B}_{\beta \beta}^{r}-\left(\mathbf{B}_{11}\right)^{2}\right\}
$$

The above inequality is equivalent to the following: $\mathscr{R} i c(W) \leq \frac{1}{2} n^{2}\|\mathbb{H}\|^{2}+\frac{\varepsilon+3}{4}(p q+n-1)-\frac{q \Delta f}{f}+\sum_{r=n+1}^{2 m+1} \sum_{\beta=2}^{n} \mathbf{B}_{11}^{r} \mathbf{B}_{\beta \beta}^{r}$.
Using (22), this gives inequality (23). For the second case, we have the following.

Case 2. Assume that $e_{u}$ is tangent $\mathbb{F}$. We fix a unit tangent vector field from $e_{p+1}, \ldots, e_{n}$ in which $W=e_{u}=e_{n}$. Utilizing (17) to (33) and following a similar technique from (33)-(42), it implies

$$
\begin{aligned}
\frac{1}{2} n^{2}\|\mathbb{H}\|^{2} \geq & \mathscr{R} i c(W)+\frac{q \Delta f}{f}-2 \widetilde{\tau}\left(T_{x} M^{n}\right)+\tilde{\tau}\left(T_{x} \mathbb{B}^{p}\right)+\tilde{\tau}\left(T_{x} \mathbb{F}^{q}\right) \\
& +\sum_{1 \leq \alpha<\beta \leq n-1} \widetilde{K}_{\alpha \beta}+\frac{1}{2} \sum_{r=n+1}^{2 m+1}\left(2 \mathbf{B}_{n n}^{r}-\left(\mathbf{B}_{p+1 p+1}^{r}+\cdots+\mathbf{B}_{n n}^{r}\right)\right)^{2} \\
& +\sum_{r=n+1}^{2 m+1} \sum_{\beta=1}^{n-1} \mathbf{B}_{n n}^{r} \mathbf{B}_{\beta \beta}^{r}+\sum_{r=n+1}^{m} \sum_{\alpha=1}^{p} \sum_{\beta=p+1}^{n}\left(\mathbf{B}_{\alpha \beta}^{r}\right)^{2}-\sum_{r=n+1}^{2 m+1} \sum_{\alpha=1}^{p} \sum_{\beta=p+1}^{n-1} \mathbf{B}_{\alpha \alpha}^{r} \mathbf{B}_{\beta \beta}^{r} .
\end{aligned}
$$


Using (34), we obtain

$$
\begin{aligned}
\mathscr{R i c}(W) \leq & \frac{1}{2} n^{2}\|\mathbb{H}\|^{2}-\frac{q \Delta f}{f}+\frac{\varepsilon+3}{4}(p q+n-1+) \\
& -\frac{1}{2} \sum_{r=n+1}^{2 m+1}\left(2 \mathbf{B}_{n n}^{r}-\left(\mathbf{B}_{p+1}^{r}+\cdots+1\right.\right. \\
& \left.\left.-\sum_{r=n+1}^{2 m+1} \sum_{\alpha=1}^{p} \sum_{\beta=p+1}^{n}\left(\mathbf{B}_{\alpha \beta}^{r}\right)^{2}+\sum_{r=n+1}^{2 m+1} \sum_{\alpha=1}^{p} \sum_{\beta=p+1}^{n-1} \mathbf{B}_{\alpha \alpha}^{r}\right)\right)^{2}-\sum_{r=n+1}^{2 m+1} \sum_{\beta=1}^{r-1} \mathbf{B}_{n n}^{r} \mathbf{B}_{\beta \beta}^{r}
\end{aligned}
$$

As the base of $M^{n}$ is minimal, then

$$
\sum_{r=n+1}^{2 m+1} \sum_{\alpha=1}^{p} \sum_{\beta=p+1}^{n-1} \mathbf{B}_{\alpha \alpha}^{r} \mathbf{B}_{\beta \beta}^{r}=0 .
$$

By using a similar technique to the first case, using (46) in (45), we obtain

$$
\begin{aligned}
\mathscr{R i c}(W) & +\frac{q \Delta f}{f} \leq \frac{1}{2} n^{2}\|\mathbb{H}\|^{2}+\frac{\varepsilon+3}{4}(p q+n-1) \\
& -\frac{1}{2} \sum_{r=n+1}^{2 m+1}\left(2 \mathbf{B}_{n n}^{r}-\left(\mathbf{B}_{p+1 q+1}^{r}+\cdots+\mathbf{B}_{n n}^{r}\right)\right)^{2}-\sum_{r=n+1}^{2 m+1} \sum_{\beta=1}^{n-1} \mathbf{B}_{n n}^{r} \mathbf{B}_{\beta \beta}^{r}-\sum_{r=n+1}^{m} \sum_{\alpha=1}^{p} \sum_{\beta=p+1}^{n}\left(\mathbf{B}_{\alpha \beta}^{r}\right)^{2} .
\end{aligned}
$$

After some calculations, we obtain

$$
\begin{aligned}
\sum_{r=n+1}^{2 m+1}\left\{\frac{1}{2}\left(\left(\mathbf{B}_{p+1 p+1}^{r}+\cdots+\mathbf{B}_{n n}^{r}\right)-2 B_{n n}^{r}\right)^{2}+\sum_{\beta=n+1}^{n-1} \mathbf{B}_{n n}^{r} \mathbf{B}_{\beta \beta}\right\} \\
=\sum_{r=n+1}^{2 m+1} \frac{1}{2}\left(\mathbf{B}_{p+1 p+1}^{r}+\cdots+\mathbf{B}_{n n}^{r}\right)^{2}+2 \sum_{r=n+1}^{m}\left(\mathbf{B}_{n n}^{r}\right)^{2} \\
\quad-\sum_{r=n+1}^{2 m+1} \sum_{\beta=p+1}^{n} \mathbf{B}_{n n}^{r} \mathbf{B}_{\beta \beta}^{r}+\sum_{r=n+1}^{m} \sum_{\beta=n+1}^{n-1} \mathbf{B}_{n n}^{r} \mathbf{B}_{\beta \beta}-\sum_{r=n+1}^{2 m+1} \sum_{\beta=p+1}^{n} \mathbf{B}_{n n}^{r} \mathbf{B}_{\beta \beta}^{r} .
\end{aligned}
$$

Performing other calculations for the last two terms gives

$$
\begin{aligned}
& \sum_{r=n+1}^{2 m+1}\left\{\frac{1}{2}\left(\left(\mathbf{B}_{p+1 p+1}^{r}+\cdots+\mathbf{B}_{n n}^{r}\right)-2 \mathbf{B}_{n n}^{r}\right)^{2}+\sum_{\beta=p+1}^{n-1} \mathbf{B}_{n n}^{r} \mathbf{B}_{\beta \beta}\right\} \\
= & \sum_{r=n+1}^{2 m+1}\left\{\frac{1}{2}\left(\mathbf{B}_{p+1 p+1}^{r}+\cdots+\mathbf{B}_{n n}^{r}\right)^{2}+\left(\mathbf{B}_{n n}^{r}\right)^{2}-\sum_{\beta=p+1}^{n-1} \mathbf{B}_{n n}^{r} \mathbf{B}_{\beta \beta}\right\} .
\end{aligned}
$$$$
\sum_{r=n+1}^{2 m+1} \sum_{\beta=n+1}^{n-1} \mathbf{B}_{n n}^{r} \mathbf{B}_{\beta \beta}-\sum_{r=n+1}^{m} \sum_{\beta=p+1}^{n} \mathbf{B}_{n n}^{r} \mathbf{B}_{\beta \beta}^{r}=-\sum_{r=n+1}^{2 m+1}\left(\mathbf{B}_{n n}^{r}\right)^{2} .
$$

Thus, (48) can be reduced, using the above relation, as follows:

Therefore, using (50) in inequality (47), we deduce that 


$$
\begin{aligned}
\mathscr{R i c}(W) \leq & \frac{1}{2} n^{2}\|\mathbb{H}\|^{2}+\frac{\mathcal{\varepsilon}+3}{4}(p q+n-1)-\frac{q \Delta f}{f}-\frac{1}{4} \sum_{r=n+1}^{2 m+1}\left(\mathbf{B}_{p+1 p+1}^{r}+\cdots+\mathbf{B}_{n n}^{r}\right)^{2} \\
& -\sum_{r=n+1}^{2 m+1}\left\{\left(\mathbf{B}_{n n}^{r}\right)^{2}-\sum_{\beta=p+1}^{n-1} \mathbf{B}_{n n}^{r} \mathbf{B}_{\beta \beta}+\frac{1}{4}\left(\mathbf{B}_{p+1 p+1}^{r}+\cdots+\mathbf{B}_{n n}^{r}\right)^{2}\right\} .
\end{aligned}
$$

From the minimality of the base of warped product submanifold $M^{n}$, we obtain

$$
\mathscr{R i c}(X) \leq \frac{1}{4} n^{2}\|\mathbb{H}\|^{2}+\frac{\varepsilon+3}{4}(p q+n-1)-\frac{q \Delta f}{f}-\sum_{r=n+1}^{2 m+1}\left(\mathbf{B}_{n n}^{r}-\sum_{\beta=p+1}^{n-1} \mathbf{B}_{n n}^{r} \mathbf{B}_{\beta \beta}\right)
$$

This gives the proof of inequality (23). We will use the technique adopted for case (i) to determine inequality (23) when $M^{n}$ is $\mathbb{F}$-minimal. Now, equality (23) can be verified in a similar manner as in $[2,3,29]$.

For a completely minimal submanifold, Proposition 1 presents the following result.
Lemma 1. Assume $\varphi: M^{n}=\mathbb{B} \times{ }_{f} \mathbb{F} \longrightarrow \tilde{M}^{2 m+1}(\varepsilon)$ is a $C$-totally real minimal isometric embedding from a warped product $M^{n}$ into a Sasakian space form $\tilde{M}^{2 m+1}(\varepsilon)$. Therefore, for any unit vector $W \in T_{x} M^{n}$, the following Ricci inequality is satisfied:

$$
\mathscr{R} i c(W)+q \Delta \ln f \leq q\|\nabla \ln f\|^{2}+\frac{\varepsilon+3}{4}(p q+n-1)
$$

where $p=\operatorname{dim} \mathbb{B}$ and $q=\operatorname{dim} \mathbb{F}$.

\section{Application to Differential Equations}

4.1. Proof of Theorem 1. Consider the following equation with $\varphi=\ln f$ :

$$
\begin{aligned}
|\operatorname{Hess}(\varphi)+t \varphi I|^{2}= & |\operatorname{Hess}(\varphi)|^{2}+t^{2}(\varphi)^{2}|I|^{2} \\
& +2 t \varphi g(\operatorname{Hess}(\varphi), I) .
\end{aligned}
$$

However, we know that $|I|^{2}=\operatorname{trace}\left(I I^{*}\right)=p$ as well as $g\left(\operatorname{Hess}(\varphi), I^{*}\right)=\operatorname{tr}\left(\operatorname{Hess}(\varphi) I^{*}\right)=\operatorname{trHess}(\varphi)$. Then, the proceeding equation takes the form

$$
|\operatorname{Hess}(\varphi)-t \varphi I|^{2}=|\operatorname{Hess}(\varphi)|^{2}+p t^{2}(\varphi)^{2}-2 t \varphi \Delta \varphi
$$

If $\lambda_{1}$ is an eigenvalue of the eigenfunction, then $\Delta \varphi=\lambda_{1} \varphi$. Thus, we obtain

$$
|\operatorname{Hess}(\varphi)+t \varphi I|^{2}=|\operatorname{Hess}(\varphi)|^{2}+\left(p t^{2}-2 t \lambda_{1}\right)(\varphi)^{2} .
$$

On the contrary, we obtain

$$
\int_{\mathbb{B} \times\{q\}} \Delta \frac{\varphi^{2}}{2} \mathrm{~d} V=\int_{\mathbb{B} \times\{q\}} \varphi \Delta \varphi \mathrm{d} V-\int_{\mathbb{B} \times\{q\}}|\nabla \varphi|^{2} \mathrm{~d} V .
$$

Again, using $\Delta \varphi=\lambda_{1} \varphi$, we have

$$
\int_{\mathbb{B} \times\{q\}}(\varphi)^{2} \mathrm{~d} V=\frac{1}{\lambda_{1}} \int_{\mathbb{B} \times\{q\}}|\nabla \varphi|^{2} \mathrm{~d} V .
$$

It follows from (56) and (58) that

$$
\int_{\mathbb{B} \times\{q\}}|\operatorname{Hess}(\varphi)+t \varphi I|^{2} \mathrm{~d} V=\int_{\mathbb{B} \times\{q\}}|\operatorname{Hess}(\varphi)|^{2} \mathrm{~d} V+\left(\frac{p t^{2}}{\lambda_{1}}-2 t\right) \int_{\mathbb{B} \times\{q\}}|\nabla \varphi|^{2} \mathrm{~d} V
$$


In particular, taking $t=\left(\lambda_{1} / p\right)$ on (59) and integrating, we obtain

$$
\int_{\mathbb{B} \times\{q\}}\left|\operatorname{Hess}(\varphi)+\frac{\lambda_{1}}{p} \varphi I\right|^{2} \mathrm{~d} V=\int_{\mathbb{B} \times\{q\}}|\operatorname{Hess}(\varphi)|^{2} \mathrm{~d} V-\frac{\lambda_{1}}{p} \int_{\mathbb{B} \times\{q\}}|\nabla \varphi|^{2} \mathrm{~d} V
$$

Again, integrating (23) and including the Green lemma, we have

$$
\int_{\mathbb{B} \times\{q\}} \mathscr{R} i c_{M}(W) \mathrm{d} V \leq \frac{n^{2}}{4} \int_{\mathbb{B} \times\{q\}}|\mathbb{H}|^{2} \mathrm{~d} V+q \int_{\mathbb{B} \times\{q\}}|\nabla \varphi|^{2} \mathrm{~d} V+\int_{\mathbb{B} \times\{q\}} \frac{\varepsilon+3}{4}(p q+n-1) \mathrm{d} V
$$

From (60) and (61), we derive

$$
\begin{aligned}
& \frac{1}{q} \int_{\mathbb{B} \times\{q\}} \mathscr{R i c}_{M}(W) \mathrm{d} V \leq \frac{n^{2}}{4 q} \int_{\mathbb{B} \times\{q\}}|\mathbb{H}|^{2} \mathrm{~d} V-\frac{p}{\lambda_{1}} \int_{\mathbb{B} \times\{q\}}\left|\operatorname{Hess}(\varphi)+\frac{\lambda_{1}}{n} \varphi I\right|^{2} \mathrm{~d} V \\
& +\frac{p}{\lambda_{1}} \int_{\mathbb{B} \times\{q\}}|\operatorname{Hess}(\varphi)|^{2} \mathrm{~d} V+\int_{\mathbb{B} \times\{q\}} \frac{\varepsilon+3}{4}\left(p+1+\frac{p-1}{q}\right) \mathrm{d} V .
\end{aligned}
$$

Under the assumption that the Ricci curvature is greater than or equal to zero, i.e., $\mathscr{R} i c(W) \geq 0$, the above equation implies

$$
\int_{\mathbb{B} \times\{q\}}\left|\operatorname{Hess}(\varphi)+\frac{\lambda_{1}}{p} \varphi I\right|^{2} \mathrm{~d} V \leq \frac{n^{2} \lambda_{1}}{4 p q} \int_{\mathbb{B} \times\{q\}}|\mathbb{H}|^{2} \mathrm{~d} V+\int_{\mathbb{B} \times\{q\}}|\operatorname{Hess}(\varphi)|^{2} \mathrm{~d} V+\frac{\lambda_{1}}{p} \int_{\mathbb{B} \times\{q\}} \frac{\varepsilon+3}{4}\left(p+1+\frac{p-1}{q}\right) \mathrm{d} V
$$

which is equivalent to the following:

$$
\int_{\mathbb{B} \times\{q\}}\left|\operatorname{Hess}(\varphi)+\frac{\lambda_{1}}{p} \varphi I\right|^{2} \mathrm{~d} V \leq \int_{\mathbb{B} \times\{q\}}|\operatorname{Hess}(\varphi)|^{2} \mathrm{~d} V+\frac{\lambda_{1}}{4 p} \int_{\mathbb{B} \times\{q\}}\left\{\frac{n^{2}}{q}|\mathbb{H}|^{2}+(\varepsilon+3)\left(p+1+\frac{p-1}{q}\right)\right\} \mathrm{d} V
$$

If the following equality holds by assumption (2),

$$
\int_{\mathbb{B} \times\{q\}}|\operatorname{Hess}(\varphi)|^{2} \mathrm{~d} V=\frac{\lambda_{1}}{4 p q} \int_{\mathbb{B} \times\{q\}}\left\{(\varepsilon+3)(1-n-p q)-n^{2}|\mathbb{H}|^{2}\right\} \mathrm{d} V
$$

then equations (64) and (65) imply that

$$
\int_{\mathbb{B} \times\{q\}}\left|\operatorname{Hess}(\varphi)+\frac{\lambda_{1}}{p} \varphi I\right|^{2} \mathrm{~d} V \leq 0 .
$$

However, it is clear that

$$
\int_{\mathbb{B} \times\{q\}}\left|\operatorname{Hess}(\varphi)+\frac{\lambda_{1}}{p} \varphi I\right|^{2} \mathrm{~d} V \geq 0 .
$$


Combining equations (66) and (67), we obtain

$$
\left|\operatorname{Hess}(\varphi)+\frac{\lambda_{1}}{n} \varphi I\right|^{2}=0 \Longrightarrow \operatorname{Hess}(\varphi)=-\frac{\lambda_{1}}{p} \varphi I
$$

Since the WF $\varphi=\ln f$ of a nontrivial WPS $M^{n}$ is a nonconstant, then equation (68), reduces to Obata's differential equation where $c=\sqrt{\lambda_{1} / p}$ by $\lambda_{1}>0$. Thus, $\mathbb{B}$ is isometric to $\mathbb{S}^{p}\left(\sqrt{\lambda_{1} / p}\right)$. This is a complete proof of the first part. On the contrary, if we have $\lambda_{1}=p$, then, from (68), we have

$$
\operatorname{Hess}(\varphi)\left(W_{1}, W_{2}\right)=-\varphi g\left(W_{1}, W_{2}\right),
$$

for any $W_{1}, W_{2} \in \Gamma(\mathbb{B})$. The proof of this theorem is completed.
4.2. Proof of Theorem 2. If $\varphi$ is the positive differential function on the Riemannian manifold $\mathbb{B}$, then Bochner formula is defined as

$$
\frac{1}{2} \Delta|\nabla \varphi|^{2}=|\operatorname{Hess}(\varphi)|^{2}+\mathscr{R} i c(\nabla \varphi, \nabla \varphi)+g(\nabla \varphi, \nabla \Delta \varphi) .
$$

Taking the integration along the volume element and using the Stokes Theorem, we obtain

$$
\int_{\mathbb{B} \times\{q\}}\left\{|\operatorname{Hess}(\varphi)|^{2}+\mathscr{R} i c(\nabla \varphi, \nabla \varphi)+g(\nabla \varphi, \nabla \Delta \varphi)\right\} \mathrm{d} V=0 .
$$

Assume that $\lambda_{1}$ is an eigenvalue of the eigenfunction; then, $\Delta \varphi=\lambda_{1} \varphi$, and we have

$$
\int_{\mathbb{B} \times\{q\}}|\operatorname{Hess}(\varphi)|^{2}=-\int_{\mathbb{B} \times\{q\}} \mathscr{R i c}(\nabla \varphi, \nabla \varphi) \mathrm{d} V-\lambda_{1} \int_{\mathbb{B} \times\{q\}}|\nabla \varphi|^{2} \mathrm{~d} V
$$

Inserting the above equation into (60), we find that

$$
\int_{\mathbb{B} \times\{q\}}\left|\operatorname{Hess}(\varphi)+\frac{\lambda_{1}}{p} \varphi I\right|^{2} \mathrm{~d} V=-\int_{\mathbb{B} \times\{q\}} \mathscr{R i c}(\nabla \varphi, \nabla \varphi) \mathrm{d} V-\lambda_{1}\left(\frac{p+1}{p}\right) \int_{\mathbb{B} \times\{q\}}|\nabla \varphi|^{2} \mathrm{~d} V .
$$

Utilizing (61) in the above equation, we arrive at

$$
\begin{aligned}
& \frac{1}{q} \int_{\mathbb{B} \times\{q\}} \operatorname{Ric}(X) \mathrm{d} V+\frac{p}{\lambda_{1}(p+2)} \int_{\mathbb{B} \times\{q\}}\left|\operatorname{Hess}(\varphi)+\frac{\lambda_{1}}{p} \varphi I\right|^{2} \mathrm{~d} V \\
& \leq \frac{n^{2}}{4 q} \int_{\mathbb{B} \times\{q\}}|\mathbb{H}|^{2} \mathrm{~d} V+\frac{(p q+n-1)}{q} \int_{\mathbb{B} \times\{q\}} \frac{\varepsilon+3}{4} \mathrm{~d} V-\frac{p}{\lambda_{1}(p+1)} \int_{\mathbb{B} \times\{q\}} \mathscr{R i c}(\nabla \varphi, \nabla \varphi) \mathrm{d} V .
\end{aligned}
$$

This can be simplified as

$$
\begin{aligned}
& \int_{\mathbb{B} \times\{q\}}\left|\operatorname{Hess}(\varphi)+\frac{\lambda_{1}}{p} \varphi I\right|^{2} \mathrm{~d} V+\frac{\lambda_{1}(p+1)}{p q} \int_{\mathbb{B} \times\{q\}} \mathscr{R i c}(X) \mathrm{d} V+\int_{\mathbb{B} \times\{q\}} \mathscr{R i c}(\nabla \varphi, \nabla \varphi) \mathrm{d} V \\
& \leq \frac{\lambda_{1} n^{2}(p+1)}{4 p q} \int_{\mathbb{B} \times\{q\}}|\mathbb{H}|^{2} \mathrm{~d} V+\frac{\lambda_{1}(p q+n-1)(p+1)}{p q} \int_{\mathbb{B} \times\{q\}} \frac{\varepsilon+3}{4} \mathrm{~d} V .
\end{aligned}
$$

Following our assumption that the Ricci curvature is greater than or equal to zero, i.e., Ric $\geq 0$, we derive that

$$
\int_{\mathbb{B} \times\{q\}}\left|\operatorname{Hess}(\varphi)+\frac{\lambda_{1}}{p} \varphi I\right|^{2} \mathrm{~d} V \leq \frac{\lambda_{1}(p+1)}{4 p q} \int_{\mathbb{B} \times\{q\}}\left\{n^{2}|\mathbb{H}|^{2}+(\varepsilon+3)(p q+n-1)\right\} \mathrm{d} V
$$


If the hypothesis of the theorem regarding the extrinsic condition (4) holds, from the above equation, we obtain

$$
\operatorname{Hess}(\varphi)\left(W_{1}, W_{2}\right)=-\frac{\lambda_{1}}{p} \varphi g\left(W_{1}, W_{2}\right),
$$

for any $W_{1}, W_{2} \in \Gamma(\mathbb{B})$. This is again Obata's ODE [1], which implies that the base $\mathbb{B}$ is isometric to the Euclidean sphere $\mathbb{S}^{p}\left(\sqrt{\lambda_{1} / p}\right)$. The proof is completed.

Using the fact that the warped product submanifold $M^{n}$ is minimal, we give the following corollary derived from Theorem 1.
Corollary 1. Let $\mathbb{M}^{2 m+1}(\varepsilon)$ be a Sasakian space form, and let $\Psi: M^{n}=\mathbb{B} \times{ }_{f} \mathbb{F} \longrightarrow \mathbb{M}^{2 m+1}(\varepsilon)$ be a $C$-totally real minimal isometric embedding of the warped product submanifold $M^{n}$ into $\mathbb{M}^{2 m+1}(\varepsilon)$ with a nonnegative Ricci curvature. Then, there is an isometry between the compact base $\mathbb{B}$ and the sphere $\mathbb{S}^{p}$ if it satisfies the following:

$$
|\operatorname{Hess}(\varphi)|^{2}=\frac{1}{4 q}\left\{(\varepsilon+3)\left(1-n-\lambda_{1} q\right)\right\} .
$$

Proof. Assuming $\Phi$ is minimal and $\lambda_{1}=p$, then, from (64), we obtain

$$
\int_{\mathbb{B} \times\{q\}}|\operatorname{Hess}(\varphi)+\varphi I|^{2} \mathrm{~d} V \leq \int_{\mathbb{B} \times\{q\}}|\operatorname{Hess}(\varphi)|^{2} \mathrm{~d} V+\frac{1}{4} \int_{\mathbb{B} \times\{q\}}(\varepsilon+3)\left(\lambda_{1}+1+\frac{\lambda_{1}-1}{q}\right) \mathrm{d} V
$$

If assumption (78) holds, we get the following from (79):

$$
\operatorname{Hess}(\varphi)\left(W_{1}, W_{2}\right)=-\varphi g\left(W_{1}, W_{2}\right),
$$

for a nonconstant function $\varphi=\ln f$. Thus, [1] completes the proof of the corollary.

Remark 3. We know that every simply connected Sasakian space form $\mathbb{M}^{2 m+1}(\varepsilon)$ is isometric to the odd-dimensional sphere $\mathbb{S}^{2 m+1}$ and odd-dimensional Euclidean space $\mathbb{R}^{2 m+1}$ with $\phi$-constant sectional curvature $\varepsilon=1$ and $\varepsilon=-3$, respectively. For more details and examples, see the work presented in $[24,26,27]$.

Another consequence of Theorem 1 is as follows.
Corollary 2. Let $\mathbb{S}^{2 m+1}$ be a Sasakian space form and $M^{n}$ be the warped product submanifold having the nonnegative Ricci curvature. If $\Psi: M^{n}=\mathbb{B} \times_{f} \mathbb{F} \longrightarrow \mathbb{S}^{2 m+1}$ is a C-totally real minimal isometric embedding of $M^{n}$ to $\mathbb{S}^{2 m+1}$. Then, there is an isometric between the compact base $\mathbb{B}$ and the sphere $\mathbb{S}^{p}$ if it satisfies the following:

$$
|\operatorname{Hess}(\varphi)|^{2}=\frac{2}{q}\left(1-n-\lambda_{1} q\right)
$$

Proof. Now, substituting $\varepsilon=1$ in (79), we derive that

$$
\int_{\mathbb{B} \times\{q\}}|\operatorname{Hess}(\varphi)+\varphi I|^{2} \mathrm{~d} V \leq \int_{\mathbb{B} \times\{q\}}|\operatorname{Hess}(\varphi)|^{2} \mathrm{~d} V+2 \int_{\mathbb{B} \times\{q\}}\left(\lambda_{1}+1+\frac{\lambda_{1}-1}{q}\right) \mathrm{d} V .
$$
obtain

Applying equation (81) into the above equation, we

$$
\operatorname{Hess}(\varphi)\left(W_{1}, W_{2}\right)=-\varphi g\left(W_{1}, W_{2}\right) \text {. }
$$

The result follows from [1]. This completes the proof of the corollary.

One result deduced from Theorem 2 is as follows.

Corollary 3. Assume that that $\mathbb{S}^{2 m+1}$ is a Sasakian space form and $M^{n}$ is a warped product submanifold having the nonnegative Ricci curvature and the compact base $\mathbb{B}$ is minimal. If $\Psi: M^{n}=\mathbb{B} \times_{f} \mathbb{F} \longrightarrow \mathbb{S}^{2 m+1}$ is a C-totally real isometric embedding from $M^{n}$ into $\mathbb{S}^{2 m+1}$, then $\mathbb{B}$ is an isometric to the sphere $\mathbb{S}^{p}(c)$ such that $c=\sqrt{\lambda_{1} / p}$ if the following equality holds:

$$
|\mathbb{H}|^{2}=\frac{4(1-p q-n)}{n^{2}}
$$

Proof. Inserting $\varepsilon=1$ into (76), we obtain

$$
\int_{\mathbb{B} \times\{q\}}\left|\operatorname{Hess}(\varphi)+\frac{\lambda_{1}}{p} \varphi I\right|^{2} \mathrm{~d} V \leq \frac{\lambda_{1}(p+2)}{4 p q} \int_{\mathbb{B} \times\{q\}}\left\{n^{2}|\mathbb{H}|^{2}+4(p q+n-1)\right\} \mathrm{d} V
$$

Following condition (84) and combining it with (85), we derive that

$$
\operatorname{Hess}(\varphi)\left(W_{1}, W_{2}\right)=-\frac{\lambda_{1}}{p} \varphi g\left(W_{1}, W_{2}\right)
$$



result.

Then, the Obata Theorem [1] leads to the desired

If we choose $\varepsilon=-3$ that is the Sasakian space form is nothing but the Euclidean space $\mathbb{R}^{2 m+1}$ by using Theorem 1 , we obtain

Corollary 4. Suppose $\Psi: M^{n}=\mathbb{B} \times_{f} \mathbb{F} \longrightarrow \mathbb{R}^{2 m+1}(-3)$ is a C-totally real isometric embedding of the warped product

$$
\int_{\mathbb{B} \times\{q\}}\left|\operatorname{Hess}(\varphi)+\frac{\lambda_{1}}{p} \varphi I\right|^{2} \mathrm{~d} V \leq \int_{\mathbb{B} \times\{q\}}|\operatorname{Hess}(\varphi)|^{2} \mathrm{~d} V+\frac{n^{2}}{4 q} \int_{\mathbb{B} \times\{q\}}|\mathbb{E}|^{2} \mathrm{~d} V .
$$

Equations (87) and (88) imply that

$$
\operatorname{Hess}(\varphi)\left(W_{1}, W_{2}\right)=-\varphi g\left(W_{1}, W_{2}\right) \text {. }
$$

Again, from Obata's Theorem [1], we reached the desired result.

Similarly, from Theorem 2, we derive

Corollary 5. Let $M^{n}$ be a warped product submanifold and $\Psi: M^{n}=\mathbb{B} \times_{f} \mathbb{F} \longrightarrow \mathbb{R}^{2 m+1}(-3)$ be a $C$-totally real minimal isometric embedding from $M^{n}$ to the Sasakian space form $\mathbb{R}^{2 m+1}(-3)$. Then, the compact base $\mathbb{B}$ is isometric to the sphere $\mathbb{S}^{p}$.

Proof. According to the hypothesis of the theorem, we know that $M^{n}$ is minimal and $\varepsilon=-3$. Therefore, from (76), we determined the ordinary differential equation (89). Thus, we have completed the proof.

Remark 4. For examples of C-totally real isometric immersions from warped product manifolds, see the work presented in $[27,30]$.

For existence such a warped product, we provided an example is the following

Example 1. (see [30]). At point $x$, there exists no less than two types C-totally real submanifolds going within $x$ of the $\operatorname{non}$-Sasakian $(\kappa, \mu)$-manifold. Assume the foliations are denoted by eigendistributions of $h$; thus, their leaves would be totally geodesic C-totally real submanifolds for the given non-Sasakian $(\kappa, \mu)$-manifold. Suppose a C-totally real submanifold in a contact metric manifold. Let us consider $g\left(A_{\zeta} X, Y\right)=-g\left(\widetilde{\nabla}_{X} \zeta, Y\right)=g(\Phi X+\Phi h X, Y)$, so $A_{\zeta}=(\Phi h)^{T}$, such that $(\Phi h)^{T} X$ is tangential part of $\Phi h X$ to any $X \in T M$. Assume that $S$ is the minimal C-totally real surface of $T_{1} M$. Construct the warped product manifold $(-(\pi / 2),(\pi /$ $2)) \times_{\cos : t} S$. Then, the embedding $\Phi:(-(\pi / 2),(\pi / 2)) \times \times_{\cos ; t}$ $S \longrightarrow T_{1} M$ such that $\Phi(t, x)=\sin t N+\cos t x$, where $N$ is the unit vector perpendicular with the linear subspace including $T_{1} M$, which is a C-totally real isometric embedding. For more classification, see [31-33]. submanifold $M^{n}$ into $\mathbb{R}^{2 m+1}(-3)$ of a nonnegative Ricci curvature. Then, a compact minimal base $\mathbb{B}$ is isometric to the sphere $\mathbb{S}^{p}$ if the following condition holds:

$$
|\operatorname{Hess}(\varphi)|^{2}+\frac{n^{2}}{4 q}|\mathbb{W}|^{2}=0 .
$$

Proof. Putting $\varepsilon=-3$ into (64), we obtain

Remark 5. We provided example 1 which shows the existence of C-totally real isometric immersion from warped product into an almost contact metric manifolds. However, we are not claiming that this example will satisfy our assumption.

\section{Concluding Remarks}

The paper dealed with ordinary differential equation on C-totally real warped product submanifolds from the optimization on the warping function of a C-totally real warped product submanifold of Sasakian space forms. First, we obtained a Ricci curvature inequality in the setting a C-totally real warped product submanifold which is a generalization of Theorem 2.1 in [24]. Then, we studied some characterizations theorems for a C-totally real warped product submanifold of a Sasakian space forms. Therefore, the paper has excellent combinations of ordinary differential equation with Riemnnian geometry. We hope that the paper will get influence in mathematical science because we first applied differential equation in product manifolds.

\section{Data Availability}

No data were used to support the findings of the study.

\section{Conflicts of Interest}

The authors declare no conflicts of interest.

\section{Authors' Contributions}

All authors equally contributed to the work and approved the final version.

\section{Acknowledgments}

This research was funded by the Deanship of Scientific Research at Princess Nourah Bint Abdulrahman University through the Fast-track Research Funding Program. 


\section{References}

[1] M. Obata, "Certain conditions for a Riemannian manifold to be isometric with a sphere," Journal of the Mathematical Society of Japan, vol. 62, pp. 333-340, 1962.

[2] A. Ali, P. Laurian-Ioan, and A. H. Alkhaldi, "Ricci curvature on warped product submanifolds in spheres with geometric applications," Journal of Geometry and Physics, vol. 146, Article ID 103510, 2019.

[3] A. Ali, P. Laurian-Ioan, A. H. Alkhaldi, and L. S. Alqahtani, "Ricci curvature on warped product submanifolds of complex space forms and its applications," International Journal of Geometric Methods in Modern Physics, vol. 16, no. 9, Article ID 1950142, 2019.

[4] R. Ali, F. Mofarreh, N. Alluhaibi, A. Ali, and I. Ahmad, "On differential equations characterizing Legendrian submanifolds of Sasakian space forms," Mathematics, vol. 8, no. 2, p. $150,2020$.

[5] H. Alodan, S. Deshmukh, N. B. Turki, and G.-E. Vîlcu, "Hypersurfaces of a sasakian manifold," Mathematics, vol. 8, no. 6 , p. $877,2020$.

[6] A. Barros, J. N. Gomes, and E. Ribeiro, "A note on rigidity of the almost Ricci soliton," Archiv der Mathematik, vol. 100, no. 5, pp. 481-490, 2013.

[7] B.-Y. Chen, "A general inequality for submanifolds in complex-space-forms and its applications," Archiv der Mathematik, vol. 67, no. 6, pp. 519-528, 1996.

[8] B.-Y. Chen, "Mean curvature and shape operator of isometric immersions in real-space-forms," Glasgow Mathematical Journal, vol. 38, no. 1, pp. 87-97, 1996.

[9] B.-Y. Chen, "Relations between Ricci curvature and shape operator for submanifolds with arbitrary codimensions," Glasgow Mathematical Journal, vol. 41, no. 1, pp. 33-41, 1999.

[10] B.-Y. Chen, "On Ricci curvature of isotropic and Lagrangian submanifolds in complex space forms," Archiv der Mathematik, vol. 74, no. 2, pp. 154-160, 2000.

[11] S. Deshmukh and A. Al-Eid, "Curvature bounds for the spectrum of a compact Riemannian manifold of constant scalar curvature," Journal of Geometric Analysis, vol. 15, no. 4, pp. 589-606, 2005.

[12] S. Deshmukh, "Conformal vector fields and eigenvectors of laplacian operator," Mathematical Physics, Analysis and Geometry, vol. 15, no. 2, pp. 163-172, 2012.

[13] S. Deshmukh and F. Al-Solamy, "A note on conformal vector fields on a Riemannian manifold," Colloquium Mathematicum, vol. 136, no. 1, pp. 65-73, 2014.

[14] S. Deshmukh, "Characterizing spheres and Euclidean spaces by conformal vector fields," Annali di Matematica Pura ed Applicata (1923 -), vol. 196, no. 6, pp. 2135-2145, 2017.

[15] S. Deshmukh, "Almost Ricci solitons isometric to spheres," International Journal of Geometric Methods in Modern Physics, vol. 16, no. 5, Article ID 1950073, 2019.

[16] F. Erkekoğlu, E. García-Río, D. N. Kupeli, and B. Ünal, "Characterizing specific Riemannian manifolds by differential equations," Acta Applicandae Mathematicae, vol. 76, no. 2, pp. 195-219, 2003.

[17] E. García-Río, D. N. Kupeli, and B. Ünal, "On a differential equation characterizing Euclidean spheres," Journal of Differential Equations, vol. 194, no. 2, pp. 287-299, 2003.

[18] S. Tanno, "Some differential equations on Riemannian manifolds," Journal of the Mathematical Society of Japan, vol. 30, no. 3, pp. 509-531, 1978.
[19] Y. Tashiro, "Complete Riemannian manifolds and some vector fields," Transactions of the American Mathematical Society, vol. 117, p. 251, 1965.

[20] A. Lichnerowicz, Geometrie des Groupes de Transformations, Dunod, Paris, France, 1958.

[21] S. Deshmukh and F. Al-Solamy, "Conformal gradient vector fields on a compact Riemannian manifold," Colloquium Mathematicum, vol. 112, no. 1, pp. 157-161, 2008.

[22] A. Ali and P. Laurian-Ioan, "Geometric classification of warped products isometrically immersed in sasakian space forms," Mathematische Nachrichten, vol. 292, pp. 234-251, 2018.

[23] J. W. Lee, C. W. Lee, and G.-E. Vîlcu, "Classification of Casorati ideal Legendrian submanifolds in Sasakian space forms," Journal of Geometry and Physics, vol. 155, Article ID 103768, 2020.

[24] I. Mihai, "Ricci curvature of submanifolds in sasakian space forms," Journal of the Australian Mathematical Society, vol. 72, no. 2, pp. 247-256, 2002.

[25] K. Yano and M. Kon, Structures on Manifolds, World Scientific, Singapore, 1984.

[26] M. I. Munteanu and L. Vrancken, "Minimal contact CR submanifolds in S2n+1 satisfying the $\delta(2)$-Chen equality," Journal of Geometry and Physics, vol. 75, pp. 92-97, 2014.

[27] T. Sasahara, "A class of biminimal legendrian submanifolds in Sasakian space forms," Mathematische Nachrichten, vol. 287, no. 1, pp. 79-90, 2014.

[28] B.-Y. Chen, "On isometric minimal immersions from warped products into real space forms," Proceedings of the Edinburgh Mathematical Society, vol. 45, no. 3, pp. 579-587, 2002.

[29] A. Mustafa, S. Uddin, and F. R. Al-Solamy, "Chen-ricci inequality for warped products in Kenmotsu space forms and its applications," Revista de la Real Academia de Ciencias Exactas, Físicas y Naturales. Serie A. Matemáticas, vol. 113, no. 4, pp. 3585-3602, 2019.

[30] M. M. Tripathi, "C-totally real warped product submanifolds," Analele Stiintifice ale Universitatii Al I Cuza din Iasi-Matematica, vol. 58, pp. 417-436, 2012.

[31] B. Y. Chen, Pseudo-Riemannian Geometry, $\delta$-invariants and Applications World Scientific, Hackensack, NJ, USA, 2011.

[32] B. Y. Chen, Differential Geometry of Warped Product Manifolds and Submanifolds, World Scientific, Singapore, 2017.

[33] A. Ali, F. Mofarreh, W. A. Mior Othman, and D. S. Patra, "Applications of differential equations to characterize the base of warped product submanifolds of cosymplectic space forms," Journal of Inequalities and Applications, vol. 2020, p. 241, 2020. 